\title{
FAIRNESS: A CHALlENGE FOR THE Distribution of CoOperation Gains in VALUe Chains
}

\author{
Stephan Zelewski and Susanne Jene \\ Institute for Production and Industrial Information Management, \\ University of Duisburg-Essen, 45141 Essen, Germany \\ stephan.zelewski@pim.uni-due.de • susanne.jene@pim.uni-due.de
}

\begin{abstract}
Most scientific publications on the subject of value chain management only analyze which structures, processes and actions can contribute to the creation of cooperation gains. How the distribution of cooperation gains that were collectively achieved can influence the stability of such a cooperation is often disregarded. The distributive justice or the fair distribution of collectively created cooperation gains is one of the most important ways to secure the stability of networks. This paper therefore presents a proposal for an operationalization of the fairness term from an economic perspective. This proposal is specific to the distribution of cooperation gains in networks of autonomously acting companies and takes a cooperative game theory approach as its basis. With the aid of the $\tau$-value, it is shown how intuitive and vague associations of fairness can be substantiated to give a concrete distribution proposal that can be perceived and communicated as fair by gradually establishing rational or at least plausible assumptions.
\end{abstract}

\section{KEYWORDS}

cooperation gains, cooperative game theory, distribution of cooperation gains, fairness, $\tau$-value, value chain management

\section{INTRODUCTION}

Value chain management is based on the idea, that, with the aid of goal-oriented management of a cooperation of multiple companies, it is possible to achieve special cooperation gains that cannot be realized without cooperation. The companies are generally assumed to be legally autonomous entities, whose cooperation does not rely on hierarchical instruction, as is the case between divisions of one company. Here it is rather implied that cooperation is based on voluntary collaboration that is economically beneficial from the perspective of each company involved. Such circumstances arise, for example, in supply chains that strictly speaking are supply webs, innovation or production networks, or virtual corporations. Such forms of inter-organizational cooperation can generally be referred to under the umbrella-term value chains because cooperation gains are achieved as added values on account of the cooperation of the actors compared to non-cooperation (defection).

The creation of cooperation gains in value chains is disputed. Basic theoretical considerations show that central coordination of collaboration based on the division of labor of several actors can never yield worse, and often yield better economic results than the aggregation of many partial plans that are locally "optimized" by each actor. However, total-planning models based on such a central coordination approach fail most of the time on account of unachievable assumptions relating to the availability of current and detailed information.

A large number of theoretical analyses and practical studies shows that cooperation gains can be achieved de facto in value chains. This also applies to complex models of multilayer supply chains, which can help to show that the mutual adjustment of the actors with regard to their 
action plans as a central coordination approach normally yields higher economic values than if the actors optimize their action plans locally and without interaction (cf. [1], [2], [3], [4], [5]). Most scientific publications on the subject of value chain management only analyze which structures, processes and actions can contribute to the cooperative creation of cooperation gains. But how the distribution of cooperation gains that were collectively achieved in a value chain can influence the stability of such a network of autonomous actors is often disregarded.

This widespread neglect of distribution aspects represents a significant research gap. The formation and the drifting apart of networks of autonomous actors generally depend on the actors regarding the distribution of hoped for or already realized cooperation gains as fair. Distributive justice, or synonymously, fair distribution of collectively created cooperation gains is one of the most important approaches to securing the stability of networks in political, socio-scientific and behavior-economic publications on the subject of network theory. Analysis of the conditions under which fair distribution of collectively created cooperation gains is realized also has very high relevance to value chain management. Without the - at least temporary - stability of cooperation in a network of autonomous actors, the cooperation gains of a value chain could not be realized in the first place.

This article therefore examines the scientific problem of how the cooperation gain can be distributed to the autonomous actors as network partners in such a way that all actors regard the distribution outcome as fair. To solve this problem, scientific methods from the area of game theory will be applied. Aspects informing the choice of methods are that the value chains considered here consist of legally independent companies (or autonomous actors for short) who each are pursuing their own interests and do not have to comply with the instructions of their cooperation partners. An analysis is additionally made from the perspective of cooperative game theory, since it is also a matter of securing the stability of the companies' cooperation by way of a perceived fair distribution of collectively achieved cooperation gains.

The relevant literature includes multiple contributions that draw on cooperative game theory to try and answer the problem of how cooperation gains that were collectively achieved in a network of autonomous actors can be distributed among the network partners in a fair way. Examples include the analyses of [6], [7], [8], [9], [10], [11], [12], [13], [14], and [15]. These contributions all share common ground in that they only cover the scientific and practical problem of fairness of cooperation gain distributions superficially. Usually, a solution concept from cooperative game theory is used, whose fairness or acceptability is implied, but not closely reflected. This applies above all for the application of the Shapley value (cf. [14], [15]), the nucleolus (e.g. [7], [15]) as well as the cooperative Nash solution (e.g. [11], [12], [13]). Only [7] discusses a wide selection of different solution concepts of cooperative game theory. He examines them mostly from a mathematical and analytical perspective, but not from the pragmatic viewpoint of their acceptability as fair solution concepts. For a detailed discussion of the current situation outlined above, see [16, pp. 30-34].

The present article discusses an innovative approach to fair distribution outcomes. It rejects the assumption of a solution concept from cooperative game theory as "a given" and its application to a distribution problem of cooperation gain while "naively" assuming that the resulting distribution outcome will be accepted as fair. Instead, it limits the space of generally possible distribution outcomes by making assumptions regarding the rationality of the actors and the measurement of their contributions to a network of autonomous actors. If all these assumptions are accepted as "plausible" or "reasonable", the result is a specific solution concept from cooperative game theory rarely found in the economic literature, the so-called $\tau$-value. The fairness of the $\tau$-value and the associated distribution outcomes is justified by the acceptability of the gradually established assumptions regarding the "plausible" or "reasonable" limitation of the 
International Journal of Managing Value and Supply Chains (IJMVSC) Vol. 2, No. 1, March 2011

valid solution space. These assumptions cannot be equated with the formalistic axioms of conventional game theory, as will be shown in the following. It is not a matter of abstract, artificial mathematical characteristics, but of intuitively understandable and, from an economic perspective, strong assumptions to a game theory concept designed to solve the above mentioned scientific - but also practical - problem of fair distribution of cooperation gains in networks of autonomous actors.

The structure of this paper is as follows: A formal language model is constructed that represents the distribution of cooperation gains in value chains as a cooperative distribution game. Within this model, the scope of possible cooperation gain distributions is gradually limited by multiple assumptions of individual and collective rationality, and of efficiency and integrity. The $\tau$-value finally results in an operational and unique game theory guideline for fair distribution outcomes. With the help of the aforementioned cooperative distribution game model, some characteristic properties of the $\tau$-value are analyzed. These properties firstly characterize the $\tau$-value as a typical compromise solution concept with some remarkable aspects. Secondly, a new interpretation of the $\tau$-value is presented from the perspective of the actors' network contributions. Thirdly, some controversial assumptions regarding the $\tau$-value are discussed. In the conclusions especially managerial insights are discussed.

\section{THE $\tau$-VALUE AND THE PROBLEM OF FAIR DISTRIBUTIONS}

\subsection{A cooperative distribution game}

The starting point for the cooperative distribution game is the generic distribution problem of distributing an cooperation gain $G$ with $G \in \mathrm{P}_{>0}$ (where $\mathrm{P}_{>0}$ is the set of all positive real numbers) among the $N$ autonomous actors $A_{n}$ of a value chain (with $n=1, \ldots, N, N \in \mathrm{N}$ and $N \geq 2$, where $\mathrm{N}$ is the set of all natural numbers). The standard approach of cooperative game theory to solving this generic distribution problem comprises two steps.

In the first step, a characteristic function $c$ is developed. This function refers to all possible coalitions which could be formed by the actors in the relevant value chain. Moreover, "degenerate" coalitions formed by one actor are feasible. Therefore, a coalition $C_{m}$ is a non-empty subset of the set $A$ of all actors in the value chain: $\varnothing \subset C_{m} \subseteq A$ with $A=\left\{A_{l}, \ldots, A_{N}\right\}$. For each characteristic function $c$, it is assumed with $\wp$ as power set operator that $c: \wp(A) \rightarrow \mathrm{P}_{\geq 0}$ with $C_{m} \rightarrow c\left(C_{m}\right)$ holds for each coalition $C_{m}$ and $\varnothing \rightarrow c(\varnothing)=0$. Such a characteristic function assigns the amount $c\left(C_{m}\right)$ the respective coalition $C_{m}$ can claim with good reasons. In the case of the grand coalition $C_{0}=A$, this is the overall cooperation gain $G: c\left(C_{0}\right)=G$. For all other coalitions $C_{m}$ with $\varnothing \subset C_{m} \subset A$, these are the amounts $c\left(C_{m}\right)$ these coalitions $C_{m}$ could realize on their own outside the grand coalition $C_{0}$ and therefore in competition with the rest of the grand coalition, i.e. the residual coalition $R C_{m}$ with $R C_{m}=C_{0} \backslash C_{m}$.

In the second step, the shape of a distribution function $v$ with $v: A \rightarrow \mathrm{P}_{\geq 0}$ and $A_{n} \rightarrow v\left(A_{n}\right)=v_{n}$ for each actor $\mathrm{A}_{\mathrm{n}}$ is determined by calculating the distribution function values $v_{n}$. Only two information sources are considered to calculate these values. On the one hand, these are the amounts which each feasible coalition $C_{m}$ can claim due to the characteristic function $c$ from the first step. On the other hand, the applied game theory solution concept specifies how the distribution function values $v_{n}$ are calculated based on the values $c\left(C_{m}\right)$ of the characteristic function $c$ for all feasible coalitions $C_{m}$ with $m=0,1, \ldots, 2^{N}-2$. When all distribution function values $v_{n}$ are determined, there is an $N$-tupel $\underline{v}=\left(v_{1}, \ldots, v_{N}\right)$ as a solution $\underline{v}$ for the respective regarded instance of the generic distribution problem. Every solution $\underline{v}$ assigns a share $v_{n}$ of the cooperation gain $G$ to each actor $A_{n}$ of the value chain. This $N$-tupel $\underline{v}$ is formally equivalent to a solution point $L$ in the $N$-dimensional non-negative real number space $\mathrm{P}_{\geq 0}{ }^{N}$. The solution point $L$ is represented as a 
column vector $\stackrel{\mathbf{l}}{v}$, whose transposed representation denoted by a superscript letter $\left.{ }^{\mathrm{T}}\right)$ is: $\stackrel{\mathrm{I}}{v}=\left(v_{1}, \ldots, v_{N}\right)^{T}$.

\subsection{The $\tau$-value as a guideline for the fair distribution of cooperation gains}

The $\tau$-value was proposed for the first time by Tijs in 1980 as part of the "Seminar on game theory and mathematical economics" [17]. It was further developed by Tijs and Driessen ([18], [19], [20], [21], [22], [23], [24]); see also [16] and [25]. Until today, only a few research projects have attempted to apply the $\tau$-value in the area of economics, especially the management of value chains. The corresponding papers, however, are focused on the distribution of fixed costs or overhead costs in single companies (cf. [24]). To the knowledge of the authors, until now relatively little research has been carried out applying the $\tau$-value to the solution of the above mentioned generic distribution problem for cooperation gains in value chains.

The standard procedure of game theory to introduce a new solution concept consists of specifying its calculation formulas and its applicability conditions. If the applicability conditions of a solution concept are available in an axiomatized form, it is often argued that the proposed solution concept is the only one that is capable of fulfilling a set of formally specified axiomatic requirements. Critical reflections on the justification of such axioms are rare. One of the drawbacks of such standard procedure is that it takes a purely formalistic approach and regards a rigorous solution concept primarily in terms of its formal calculation and, if possible, its formal axiomatization. From a management point of view, it lacks orientation towards the real problem of a distribution outcome being accepted as fair.

The following procedure therefore sets out to justify a game theory solution in an alternative line of reasoning: Starting from the real problem of distributing cooperation gains among the actors of a value chain, the solution concept should be developed in an easily understandable manner so that it is derivable from "plausible" or "reasonable" assumptions oriented towards the real problem under consideration. Moreover, it should be possible to have or to give good reasons for accepting the resulting solution as fair distribution. In the following, the authors try to develop a generally applicable justification program for game theory solution concepts oriented towards real problems of cooperation gain distribution as exemplified by the specific $\tau$-value solution concept. A major concern of this paper is to reconstruct the $\tau$-value solution concept in a new way with regard to this justification program.

The basic idea of the reconstruction of the $\tau$-value solution concept is to restrict the solution space $\mathrm{P}_{\geq 0}{ }^{N}$ for the generic distribution problem by successively adding five assumptions which stem from the real problem of distributing cooperation gains achieved cooperatively in a value chain among the cooperating actors.

The first assumption is the condition of individual rationality. This condition assumes that every actor in a value chain acts rationally in the sense of the conventional concept of perfect rationality. This causes a restriction of the solution space $\mathrm{P}_{\geq 0}{ }^{N}$, since it would not be rational for an actor $A_{n}$ to participate in the value chain within the grand coalition $C_{0}$ if this coalition yields a smaller utility for this actor compared to his or her leaving the coalition and realizing the amount $c\left(\left\{A_{n}\right\}\right)$ outside the value chain. Thus the condition of individual rationality can be formulated with the characteristic function $c$ and the feasible solution point $L$ within the solution space as follows:

$\forall L \in i_{i} \stackrel{N}{\geq 0}: \quad L=\left(v_{1}, \ldots v_{N}\right)^{T} \geq\left(c\left(\left\{A_{1}\right\}\right), \ldots, c\left(\left\{A_{N}\right\}\right)\right)^{T}$

The second assumption is the efficiency condition. This condition postulates that the cooperation gain $G$ is distributed exactly ("efficiently") among all actors $A_{n}$ of the grand coalition 
$C_{0}=\left\{\mathrm{A}_{1}, \ldots, \mathrm{A}_{\mathrm{N}}\right\}$. On the one hand, it would be irrational to distribute less than the cooperation gain $G$, because this would necessarily entail a loss of Pareto optimality. On the other hand, it would be impossible to distribute more than the cooperation gain $G$. Thus the following equation will hold:

$$
\forall L \in i_{\geq 0}^{N}: \quad L=\left(v_{1}, \ldots v_{N}\right)^{T} \rightarrow \sum_{n=1}^{N} v_{n}=c\left(C_{0}\right)=G
$$

The efficiency condition implies a further restriction of the solution space $\mathrm{P}_{\geq 0}{ }^{N}$, since all the solutions of the distribution problem that fulfill the assumption of individual rationality as well as the assumption of efficiency are solution points $L$ on a hyperplane $H$ in the $N$-dimensional solution space $\mathrm{P}_{\geq 0}{ }^{N}$.

The third assumption is the rationality condition for maximum allocable shares of the cooperation gain. This condition has the character of a condition of collective rationality, since it mirrors the rational consideration of all $N-1$ actors of the marginal coalition $M C_{n}$ with $M C_{n}=$ $C_{0} \backslash\left\{A_{n}\right\}=\left\{A_{1}, \ldots, A_{n-1}, A_{n+1, \ldots}, A_{N}\right\}$ to grant actor $A_{n}$ at most the share $v_{n . \max }$ of the cooperation gain $G$, so that the cooperation gain would decrease if actor $A_{n}$ left the grand coalition $C_{0}=$ $\left\{A_{1}, \ldots, A_{N}\right\}$. This rationality condition requires the following where $c\left(C_{0}\right)=G$ holds from formula (2.2.2):

$$
\forall n=1, \ldots, N \forall v_{n} \in \mathrm{i}_{\geq 0}: v_{n} \leq v_{n \cdot \max } \wedge v_{n \cdot \max }=c\left(C_{0}\right)-c\left(M C_{n}\right)=G-c\left(M C_{n}\right)
$$

In the solution space, the point at which the maximum allocable share $v_{n \text { max }}$ of the cooperation gain $G$ is assigned to each actor $A_{n}$, is called the upper bound $U B$ or ideal point for the distribution of an cooperation gain $G$.

The fourth assumption is a rationality condition for minimum allocable shares of the cooperation gain. This condition also has the character of a condition of collective rationality, since the condition reflects the rational consideration of all $N-1$ actors of the marginal coalition $M C_{n}$ with $M C_{n}=C_{0} \backslash\left\{A_{n}\right\}$ to grant actor $A_{n}$ at least the share $v_{n . \min }$ of the cooperation gain $G$ with which she or he could credibly threaten to found at least one outsider coalition. An outsider coalition is a coalition $A C_{n . q}$ of former actors of the value chain, which leaves the grand coalition $C_{0}$, at least hypothetically, and has at least the actor $A_{n}$ as "leader". Since the same actor $A_{n}$ can lead several outsider coalitions, the second index $q$ is used to differentiate all outsider coalitions led by the same actor $A_{n}$. Furthermore, an outsider coalition can never contain all actors of the grand coalition $C_{0}$, since no non-empty residual coalition would exist whose actors could generate the cooperation gain $G$ to be distributed.

For the $\tau$-value, it is important which outsider coalitions $A C_{n . q}$ enable an actor $A_{n}$ to threaten in a believable manner. In this paper, it is assumed that the characteristic function is partially known due to the amounts $\mathrm{c}\left(A C_{n . q}\right)$ for each outsider coalition led by an actor $A_{n}$. The actor $A_{n}$ offers all other actors of the outsider coalition $A C_{n . q}$ an optimal incentive to defect. This incentive consists of so-called side payments and ensures that the utility of each other actor out of the considered outsider coalition $A C_{n . q}$ is the same as his or her maximum utility in the grand coalition $C_{0}$. In this case, the actors in an outsider coalition have no incentive to remain in the grand coalition $C_{0}$. The operationalization of the side payments takes place in the following way, with the amount $c\left(\left\{A_{n}\right\} \mid A C_{n . q}\right)$ realizable by actor $A_{n}$ in the outsider coalition $A C_{n . q}$ and with the index set $I N_{n . q}$ of indices of all actors belonging to this outsider coalition:

$$
\begin{aligned}
\forall \varnothing \subset A C_{n \cdot q} \subset A: & \left\{A_{n}\right\} \subset A C_{n . q} \rightarrow \ldots \\
& c\left(\left\{A_{n}\right\} \mid A C_{n . q}\right)=c\left(A C_{n \cdot q}\right)-\sum_{m \in\left(I N_{n \cdot q} \backslash\{n\}\right)} v_{m . \max }
\end{aligned}
$$


The amounts $c\left(\left\{A_{n}\right\} \mid A C_{n . q}\right)$ utilized by actor $A_{n}$ in threatening to found an outsider coalition may be negative. In this case a threat would not be believable. Thus this case is excluded from the rationality condition for minimum allocable shares $v_{n \text {.min }}$ of the cooperation gain $G$. The complete rationality condition is as follows:

$\forall n=1, \ldots, N \forall v_{n} \in \mathrm{i}_{\geq 0}: v_{n} \geq v_{n . \min } \wedge v_{n . \min }=\max \left\{c_{n .1} ; c_{n .2} ; 0\right\}$

with:

$$
\begin{aligned}
& c_{n .1}=c\left(\left\{A_{n}\right\} \mid A C_{n . q}\right)=c\left(\left\{A_{n}\right\}\right) \text { for } A C_{n . q}=\left\{A_{n}\right\} \\
& c_{n .2}=\max \left\{\begin{array}{c}
c\left(\left\{A_{n}\right\} \mid A C_{n . q}\right)=c\left(A C_{n . q}\right)-\sum_{m \in\left(N_{n . q} \backslash\{n\}\right)} v_{m . \max } \mid \cdots \\
\varnothing \subset A C_{n . q} \subset A \wedge\left\{A_{n}\right\} \subset A C_{n . q}
\end{array}\right\}
\end{aligned}
$$

The lower bound $L B$ for the distribution of the cooperation gain $G$ is that point in the solution space $\mathrm{P}_{\geq 0}{ }^{N}$ in which the minimum allocable shares $v_{n \text {.min }}$ of the cooperation gain $G$ are assigned to each actor $A_{n}$ where $n=1, \ldots, N$. The lower bound $L B$ is often called the threat point (e.g. [26]); in the $\tau$-value literature especially, it is called the minimal right vector (e.g. [17], [27]).

The fifth and last assumption is introduced as an integrity condition for the relation of the lower bound $L B$ to the upper bound $U B$ for the shares of the cooperation gain $G$ to be distributed, as well as for the hyperplane $H$ for the compliance with the efficiency condition to avoid particular complications outside the scope of this paper (for details of these complications see [16, pp. 137-141 and 156-167]). Games which satisfy this integrity condition are designated quasibalanced games in the game theory literature:

$$
\begin{aligned}
& \forall L B, U B \in \mathrm{i}_{\geq 0}^{N} \forall G \in \mathrm{i}>0: \\
& \left(L B=\left(\begin{array}{c}
v_{1 . \text { min }} \\
\cdots \\
v_{N . \text { min }}
\end{array}\right) \wedge U B=\left(\begin{array}{c}
v_{1 . \text { max }} \\
\cdots \\
v_{N \cdot \max }
\end{array}\right) \wedge c\left(C_{0}\right)=G\right) \\
& \rightarrow\left(\sum_{n=1}^{N} v_{n . \text { min }} \leq G \leq \sum_{n=1}^{N} v_{n \cdot \max } \wedge L B \leq U B\right)
\end{aligned}
$$

It can be shown [16, pp. 153-163] that exactly one solution point $\mathrm{L}$ exists in the $\mathrm{N}$-dimensional non-negative real number space $\mathrm{P}_{\geq 0}{ }^{N}$ that fulfills all five aforementioned assumptions for the generic distribution problem concerning individual and collective rationality as well as efficiency and integrity, i.e. the formulas (2.2.1), (2.2.2), (2.2.3), (2.2.5), and (2.2.6). This unique solution point is the $\tau$-value. The $\tau$-value is a special solution point $L_{\tau}$ which is determined by a convex - or to put it in less precise but more intuitive terms: linear - combination of the upper bound (ideal point) $U B$ and the lower bound (threat point) $L B$ with the weighting factor $\gamma$ and $0 \leq \gamma \leq 1$. Therefore it must hold true that:

$$
\begin{aligned}
& \forall L, L B, U B \in \mathbb{R}_{20}^{N} \forall G \in \mathbb{R}_{>0}: \\
& \left(\begin{array}{l}
L=\left(\begin{array}{c}
v_{1} \\
\ldots \\
v_{N}
\end{array}\right) \wedge \sum_{n=1}^{N} v_{n}=G \wedge L B=\left(\begin{array}{c}
v_{1, \min } \\
\ldots \\
v_{N . \text { min }}
\end{array}\right) \wedge U B=\left(\begin{array}{c}
v_{1, \max } \\
\ldots \\
v_{N \cdot \text { max }}
\end{array}\right) \\
\wedge \sum_{n=1}^{N} v_{n, \min } \leq G \leq \sum_{n=1}^{N} v_{n, \max } \wedge L B \leq U B
\end{array}\right)
\end{aligned}
$$

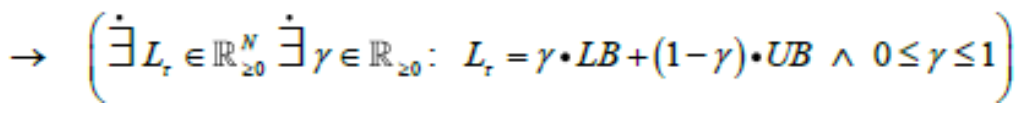


In its most widespread representation in literature (e.g. [22], [25], [28]), the $\tau$-value is explicitly specified for each actor $A_{n}$. After some simple transformations using the efficiency condition and with special regard to the frequently ignored degenerated case $\sum_{n=1}^{N} v_{n \text {.max }}=\sum_{n=1}^{N} v_{n \text {.min }}$, the common formula for calculating the $\tau$-value produces:

$\forall n=1, \ldots, N: \quad v_{n, \tau}=\gamma \mathrm{g} v_{n, \max }+(1-\gamma) \mathrm{g} v_{n, \min }$

with:

$$
\begin{array}{ll}
\gamma=\frac{G-\sum_{n=1}^{N} v_{n . \min }}{\sum_{n=1}^{N} v_{n, \max }-\sum_{n=1}^{N} v_{n . \min }} ; & \text { if } \sum_{n=1}^{N} v_{n . \max } \neq \sum_{n=1}^{N} v_{n . \min } \\
\gamma \in[0 ; 1] ; & \text { if } \sum_{n=1}^{N} v_{n . \max }=\sum_{n=1}^{N} v_{n . \min }
\end{array}
$$

\section{MODEL-ANALYTIC CHARACTERIZATION AND CRITIQUE OF THE $\tau$-VALUE}

\subsection{Characteristic properties of the $\tau$-value}

The $\tau$-value according to equation (2.2.8) shows that the $\tau$-value is to be considered as a typical compromise solution for each actor $A_{n}$ between its maximum allocable share $v_{n \text {.max }}$ (i.e. the $n$-th component of the ideal point $U B$ ) and its minimum allocable share $v_{n \text {.min }}$ (i.e. the $n$-th component of the threat point $L B$ ) of the cooperation gain $G$. The characterization of the $\tau$-value as a compromise solution for the generic distribution problem presents another good reason to accept the distribution of the of the cooperation gain as fair, since intuitive preconceptions about what is accepted as fair contain the normative connotation that fair distribution should be based on a compromise between the interests of the involved actors. In the case of the $\tau$-value solution concept, these interests are operationally specified with the aid of the threat point and the ideal point.

If the efficiency condition is additionally taken into account, allowing the weighting factor $\gamma$ to be specified numerically, it is possible to represent the $\tau$-value in a very easily intelligible manner as the convex combination of the upper bound $U B$ (ideal point) and the lower bound $L B$ (threat point) lying on the hyperplane $H$ within the $N$-dimensional solution space $\mathrm{P}_{\geq 0}{ }^{N}$ and therefore satisfying the efficiency condition. This is illustrated in Figure 1 below for the special case of three actors, i.e. $N=3$ : 


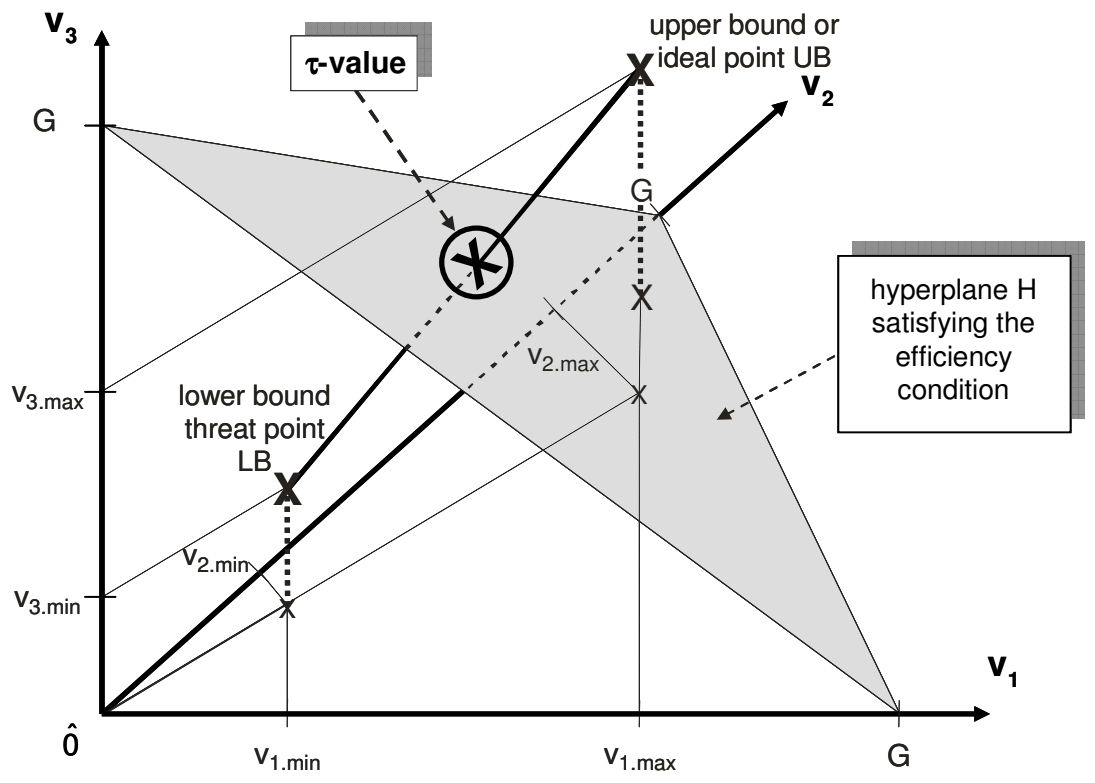

Figure 1. Illustration of the $\tau$-value

The $\tau$-value is not only a typical compromise solution but is also characterized by two additional characteristic properties. Firstly, the compromise solution is Pareto efficient in terms of the above mentioned efficiency condition. Thus the $\tau$-value can be understood as an efficient compromise. Secondly, the compromise solution represents the intuitively simplest compromise between the threat point and the ideal point. It is impossible to construct a simpler connection between these two points in the solution space $\mathrm{P}_{\geq 0}{ }^{N}$ than the direct rectilinear distance determined by equations (2.2.8) and (2.2.9).

\subsection{A new interpretation of the $\tau$-value}

A new interpretation of the $\tau$-value is given from the perspective of the actors cooperating in the network of a value chain. This interpretation clearly differs from the description of the $\tau$-value in the game theory literature. But it reveals the economic content of the $\tau$-value more because it ties in with the scope of possible actions that empower an actor to contribute to the realization of cooperation gains in a value chain - both in a positive and a negative way.

It is required as a new assumption that solution concepts for the generic distribution problem fulfill an operational fairness criterion: the greater the bargaining power of an actor $A_{n}$, the greater his or her share $v_{n . \tau}$ of the cooperation gain $G$. The bargaining power of an actor $A_{n}$ depends on two opposed effects. On the one hand, the bargaining power of an actor $A_{n}$ is measured by the contribution the actor would make if she or he took part in the marginal coalition $M C_{n}$ and thus would make this marginal coalition a grand coalition $C_{0}$. This positive network effect is the maximum allocable share $v_{n \text {.max }}$ of the cooperation gain; see formula (2.2.3). On the other hand, the bargaining power of an actor $A_{n}$ is measured by his or her threat potential that is buildup of the believable threat to found at least one outsider coalition $A C_{n . q}$. This negative network effect has been specified as the minimum allocable share $v_{n \text {.min }}$ of the cooperation gain; see formula (2.2.5). 
The good reasons for accepting the distribution of the cooperation gain $G$ among the actors $A_{n}$ in a value chain, and therefore a feasible solution $L$ of the generic distribution problem as fair, can be specified as follows: It is regarded as fair to grant actor $A_{n}$ a share $v_{n}$ of the cooperation gain $G$ which is positively correlated with the actor's contribution to building the grand coalition (positive network effect) and with the threat potential to preventing the grand coalition from coming into existence (negative network effect).

The aforementioned characterization of a fair distribution of the cooperation gain $G$ is mainly qualitative. Thus, this characterization offers some scope for interpretation regarding the numerical determination of the shares $v_{n}$ for all actors $A_{n}$ in a value chain. A quantification of the fairness criterion in the form of a calculation rule for the $\tau$-value is desirable. This calculation rule should be as easy as possible to understand in order to gain acceptance in management practice. The calculation rule employed is the following new type of formula to calculate the $\tau$ value:

$$
\begin{aligned}
& \forall n=1, \ldots, N: \\
& v_{n, r}= \begin{cases}\alpha \cdot \frac{v_{n \cdot \max }}{\sum_{n=1}^{N} v_{n \cdot \max }} \cdot G+\beta \cdot \frac{v_{n \cdot \min }}{\sum_{n=1}^{N} v_{n, \min }} \cdot G ; & \text { if } \sum_{n=1}^{N} v_{n \cdot \max } \neq \sum_{n=1}^{N} v_{n \cdot \min } \\
v_{n, \max }=v_{n \cdot \min } ; & \text { if } \sum_{n=1}^{N} v_{n \cdot \max }=\sum_{n=1}^{N} v_{n \text { min }}\end{cases}
\end{aligned}
$$

with:

$$
\begin{array}{r}
\alpha=\frac{G-\sum_{n=1}^{N} v_{n, \min }}{\sum_{n=1}^{N} v_{n, \max }-\sum_{n=1}^{N} v_{n, \min }} \cdot \frac{\sum_{n=1}^{N} v_{n, \max }}{G} \\
\wedge \quad \beta=\frac{\sum_{n=1}^{N} v_{n \cdot \max }-G}{\sum_{n=1}^{N} v_{n, \max }-\sum_{n=1}^{N} v_{n \cdot \min }} \cdot \frac{\sum_{n=1}^{N} v_{n \cdot \min }}{G}
\end{array}
$$

Three properties of the new formula type are particularly remarkable. Firstly, this calculation rule is characterized by capturing the bargaining power of the actor $A_{n}$ by two summands. The first summand reflects the bargaining power of the actor according to his or her contribution to the grand coalition (positive network effect or coalition contribution) by means of the share $v_{n \text { max }}$ of the cooperation gain $G$. The second summand represents the bargaining power of the actor due to his or her threat potential to dissolve the grand coalition (negative network effect or threat potential) by means of the share $v_{n \text {.min }}$ of the cooperation gain $G$. Secondly, the coalition contribution and the threat potential of actor $A_{n}$ are not measured absolutely, but are relativized with respect to the sums of the coalition contributions and the threat potentials respectively. This is a normalization of the coalition contribution and of the threat potential of actor $A_{n}$ regarding the upper bound $U B$ and the lower bound $L B$ respectively. Thirdly, the coalition contribution and the threat potential of actor $A_{\mathrm{n}}$ are weighted with the factors $\alpha$ and $\beta$ respectively. These weighting factors connect the share $v_{n}$ of the cooperation gain $G$ that an actor $A_{\mathrm{n}}$ receives in a typical proportional manner with the two central aspects of bargaining power, the coalition contribution $v_{n \text {.max }}$ and the threat potential $v_{n \text {.min }}$. This property of proportionality plays an important role for the acceptability of the $\tau$-value, because proportionality is frequently assumed as a plausible and convincing essence of fairness. However, the proportionality between the share $v_{n}$ of the cooperation gain $G$ on the one side and the coalition contribution and the threat potential on the other side does not apply exactly, but only in a rough approximation (for details see [16, pp. 184-189 and 224-230]). 
For interested readers, it is shown that both sets of formulas for the calculation of the $\tau$-value the formulas (2.2.8) and (2.2.9) popular in the game theory literature on the one hand and the new formulas (3.2.1) and (3.2.2) presented here on the other hand - are equivalent from a mathematical point of view.

In the game theory literature, the $\tau$-value according to equation (2.2.8) is usually regarded with respect to each actor $A_{n}$ as a convex combination of his or her coalition contribution $v_{n \cdot \max }$ (as a component of the ideal point $U B$ ) and his or her threat potential $v_{n \text {.min }}$ (as a component of the threat point $L B$ ) with the weighting factor $\gamma$ and $0 \leq \gamma \leq 1$ :

$$
\forall n=1, \ldots, N: \quad v_{n, \mathrm{r}}=\gamma \cdot v_{n, \max }+(1-\gamma) \cdot v_{n \text {.min }}
$$

In consideration of the efficiency condition according to formula (2.2.2), the formula (2.2.9) for the $\tau$-value follows:

$$
\begin{gathered}
\forall n=1, \ldots, N: v_{n, x}=\gamma \cdot v_{n \cdot \max }+(1-\gamma) \cdot v_{n \cdot \min } \\
\wedge \sum_{n=1}^{N} v_{n, r}=G \\
\Leftrightarrow \sum_{n=1}^{N}\left(\gamma \cdot v_{n \cdot \max }+(1-\gamma) \cdot v_{n \cdot \min }\right)=G \\
\Leftrightarrow \gamma=\frac{G-\sum_{n=1}^{N} v_{n \cdot \min }}{\sum_{n=1}^{N} v_{n \cdot \max }-\sum_{n=1}^{N} v_{n \cdot \min }} ; \text { if } \sum_{n=1}^{N} v_{n \cdot \max } \neq \sum_{n=1}^{N} v_{n \cdot \min } \\
\text { Standard case: } \sum_{n=1}^{N} v_{n \cdot \max } \neq \sum_{n=1}^{N} v_{n \cdot \min }
\end{gathered}
$$

By representing the $\tau$-value for all involved actors $A_{n}$ with $n=1, \ldots, N$ as a solution point $L_{\tau}$ in the $N$-dimensional solution space $\mathrm{P}_{\geq 0}{ }^{N}$, it applies that:

$$
\begin{aligned}
& L_{\tau}=\gamma \cdot\left(\begin{array}{c}
v_{1, \max } \\
\ldots \\
v_{N \cdot \max }
\end{array}\right)+(1-\gamma) \cdot\left(\begin{array}{c}
v_{1, \min } \\
\ldots \\
v_{N \cdot \min }
\end{array}\right) \wedge \gamma=\frac{G-\sum_{n=1}^{N} v_{n, \min }}{\sum_{n=1}^{N} v_{n \cdot \max }-\sum_{n=1}^{N} v_{n \cdot \min }} \\
& \Leftrightarrow L_{\tau}=\frac{G-\sum_{n=1}^{N} v_{n \cdot \min }}{\sum_{n=1}^{N} v_{n, \max }-\sum_{n=1}^{N} v_{n, \min }} \cdot\left(\begin{array}{c}
v_{1 \max } \\
\cdots \\
v_{N \cdot \max }
\end{array}\right) \\
& +\left(1-\frac{G-\sum_{n=1}^{N} v_{n \cdot \min }}{\sum_{n=1}^{N} v_{n \cdot \max }-\sum_{n=1}^{N} v_{n, \min }}\right) \cdot\left(\begin{array}{c}
v_{1, \min } \\
\ldots \\
v_{N-\min }
\end{array}\right)
\end{aligned}
$$




$$
\begin{aligned}
& \Leftrightarrow L_{r}=\frac{G-\sum_{n=1}^{N} v_{n \cdot \min }}{\sum_{n=1}^{N} v_{n \cdot \max }-\sum_{n=1}^{N} v_{n \cdot \min }} \cdot \frac{\sum_{n=1}^{N} v_{n \cdot \max }}{G} \cdot\left(\begin{array}{c}
\frac{v_{1, \max }}{\sum_{n=1}^{N} v_{n \cdot \max }} \cdot G \\
\ldots \\
\frac{v_{N \cdot \max }}{\sum_{n=1}^{N} v_{n \cdot \max }} \cdot G
\end{array}\right) \\
& +\frac{\sum_{n=1}^{N} v_{n, \max }-G}{\sum_{n=1}^{N} v_{n \text { max }}-\sum_{n=1}^{N} v_{n, \min }} \cdot \frac{\sum_{n=1}^{N} v_{n, \min }}{G} \cdot\left(\begin{array}{c}
\frac{v_{1, \min }}{\sum_{n=1}^{N} v_{n, \min }} \cdot G \\
\ldots \\
\frac{v_{N \cdot \min }}{\sum_{n=1}^{N} v_{n, \min }} \cdot G
\end{array}\right)
\end{aligned}
$$

Definitional introduction of the weighting factors $\alpha$ and $\beta$ in formula (3.2.2) allows the equation (3.2.7) to be represented in a simplified but equivalent form:

$$
L_{\tau}=\alpha \cdot\left(\begin{array}{c}
v_{1 \max } \\
\ldots \\
v_{N \cdot \max }
\end{array}\right) \cdot \frac{G}{\sum_{n=1}^{N} v_{n \cdot \max }}+\beta \cdot\left(\begin{array}{c}
v_{1 \cdot \min } \\
\ldots \\
v_{N \cdot \min }
\end{array}\right) \cdot \frac{G}{\sum_{n=1}^{N} v_{n \cdot \min n}}
$$

For the components $v_{n . \tau}$ of the $\tau$-value $\underline{v}_{\tau}$ and the corresponding solution point $L_{\tau}$ in the solution space $\mathrm{P}_{\geq 0}{ }^{N}$, it immediately follows that:

$$
\forall n=1, \ldots, N: \quad v_{n, \tau}=\alpha \cdot \frac{v_{n \max }}{\sum_{n=1}^{N} v_{n \cdot \max }} \cdot G+\beta \cdot \frac{v_{n \cdot \min }}{\sum_{n=1}^{N} v_{n \cdot \min }} \cdot G
$$

Formula (3.2.9) conforms to equation (3.2.1) with respect to the standard case.

Special case: $\sum_{n=1}^{N} v_{n \cdot \max }=\sum_{n=1}^{N} v_{n \cdot \min }$

In this special case, the upper bound $U B$ and the lower bound $L B$ have to be the same $(U B=L B)$ with respect to the fulfillment of the integrity condition of formula (2.2.6) and with respect to $v: A \rightarrow \mathrm{P}_{\geq 0}$ for every distribution function $v$. Therefore it holds $v_{n \cdot \min }=v_{n \cdot \max }$ for each actor $A_{n}$ with $n=1, \ldots, N$. Thus the upper bound $U B$ and the lower bound $L B$ lie on the same point on the hyperplane $H$ in the solution space $\mathrm{P}_{\geq 0}{ }^{N}$. This means for the representation of the $\tau$-value as a convex combination of the ideal point (the upper bound $U B$ ) and the threat point (the lower bound $L B$ ) according to equation (2.2.9) that for the weighting factor $\gamma$ with $0 \leq \gamma \leq 1$ each value $\gamma$ out of the real-valued interval $[0 ; 1]$ can be chosen arbitrarily based on the coincidence of the ideal point and the threat point:

$$
\begin{aligned}
& \forall n=1 \ldots . . N \forall v \in[0: 1] M \text { : } \\
& v_{n, r}=\gamma \cdot v_{n \text { max }}+(1-\gamma) \cdot v_{n \text { min }} \wedge v_{n \text { min }}=v_{n \text { max }}=v_{n, t} \\
& \rightarrow v_{n, r}=\gamma \cdot v_{n . t}+(1-\gamma) \cdot v_{n . r} \\
& \rightarrow v_{n . r}=\gamma \cdot v_{n . t}+v_{n . t}-\gamma \cdot v_{n . r}=v_{n . r} \quad \text { true for every } \gamma \in[0 ; 1]
\end{aligned}
$$


Special case: $\sum_{n=1}^{N} v_{n \cdot \max } \leq \sum_{n=1}^{N} v_{n \cdot \min }$ with $v_{n \cdot \max }<v_{n \cdot \min }$ for at least one actor $A_{n}$. This case is ruled out by the integrity condition according to formula (2.2.6). It therefore requires no further consideration.

\subsection{Controversial aspects of the $\tau$-value}

The inquiry presented here represents the thesis that the $\tau$-value as a game theory solution concept for the above mentioned generic distribution problem is an interesting approach to operationalizing the generally only vague conceptions regarding the fairness of distribution outcomes. Basically, there are three objections of varying importance to this thesis.

Firstly, good arguments exist for and against the question of whether it is "adequate" to apply the operationalization of fairness only to the bargaining power of each actor $A_{n}$. Furthermore, it is also possible to question whether it is "adequate" to measure this bargaining power by the actor's contribution to the grand coalition (positive network effect) and by the actor's threat potential to dissolve the grand coalition (negative network effect). This criticism relates to both formulas (3.2.1) and (3.2.2) as a calculation rule for the $\tau$-value.

This first objection against the $\tau$-value is relatively weak compared to the two following objections. Both formulas (3.2.1) and (3.2.2) are not at all constitutive for the $\tau$-value. Furthermore, they are entirely dispensable because the $\tau$-value, as shown in Chapter 2.2, inevitably results only from the five assumptions according to the formulas (2.2.1), (2.2.2), (2.2.3), (2.2.5) and (2.2.6) without recourse to those two formulas (3.2.1) and (3.2.2). The formulas (3.2.1) and (3.2.2) only represent an equivalent representation of the usual representation of the $\tau$-value with the help of the two formulas (2.2.8) and (2.2.9). However, this equivalent representation has the advantage that the solution concept of the $\tau$-value can be interpreted very strongly from an economic perspective. Additionally, there is an immanent weakness in this critical scepticism as to whether the operationalization of fairness with the aid of both formulas (3.2.1) and (3.2.2) is "adequate", because it doubts the adequacy of a distinct solution concept without expanding on the relevant adequacy criteria. As long as this criterion of adequacy is not explained, the criticism of the apparent inadequacy of a specific solution concept has no basis. On the contrary, it can be seen as a special asset of the $\tau$-value that it makes a concrete discussion proposition for the operationalization of fairness, which may lead to alternative, but equally concrete, operationalization propositions.

Secondly, the assumption of collective rationality concerning the minimum allocable shares of the cooperation gain represents a very critical aspect of the $\tau$-value. This assumption is based on an operationalization of the meaning of a "believable" threat. The credibility of a threat is always a matter of discretion that depends on subjective evaluations and for that reason can always be questioned - from the perspective of other equally subjective evaluations. Hence the solution concept of the $\tau$-value has a weakness in this area. Operationalization of the meaning of a "believable" threat with outsider coalitions only needs to be questioned to undermine the whole solution concept; see for example [7, p. 187]. To eliminate this weakness as effectively as possible, the solution concept of the $\tau$-value conceptualizes the credibility of threats with outsider coalitions in a particularly rigid way. This was explained in detail in association with the formula (2.2.5) in chapter 2.2.

Thirdly, the assumption of the integrity condition - see formula (2.2.6) for the restriction to the class of quasi-balanced games - is a problem. It was introduced in chapter 2.2 in relation to avoiding particular complications outside the scope of this paper. Without discussing the details of these complications, the meaning of the integrity condition of formula (2.2.6) for the solution concept of the $\tau$-value can be described as following. Without this integrity condition, the first four assumptions according to formulas (2.2.1), (2.2.2), (2.2.3) and (2.2.5) can only prove the 
International Journal of Managing Value and Supply Chains (IJMVSC) Vol. 2, No. 1, March 2011

existence of at most one solution point $L$ in the $N$-dimensional non-negative real number space $\mathrm{P}_{\geq 0}{ }^{N}$ that fulfills these four assumptions. But it does not assure the existence of at least one solution point $L$ at the same time. This is because "pathological" cases exist, which do not satisfy the formula (2.2.6). In these "pathological" cases there is no solution concept that satisfies the four assumptions according to the formulas (2.2.1), (2.2.2), (2.2.3) and (2.2.5) at the same time. Thus the $\tau$-value does not exist in these cases. Only the addition of the integrity condition of formula (2.2.6) ensures that not only a minimum, but also a maximum of one solution point $L$ exists at the same time in the $N$-dimensional non-negative real number space $\mathrm{P}_{\geq 0}{ }^{N}$ that fulfills the five assumptions. This exact one single solution point $L$ is the $\tau$-value, as shown above.

The limitation of the $\tau$-value to quasi-balanced games can be seen as a substantial weakness of the $\tau$-value. But a weakness would only exist if theoretically interesting or practically relevant instances of the generic distribution game that do not belong to the class of quasi-balanced games were specified. This has not been the case up to now. In fact, it can be shown [16, pp. 163-166] that those instances of the generic distribution game not belonging to the class of quasi-balanced games are not relevant to real problems of the distribution of a cooperation gain.

\subsection{A practical example for calculating the $\tau$-value}

In the following, a numerical example shows how the $\tau$-value can be applied concretely in management practice to solve the problem of fair distribution of cooperation gains in value chains. For illustrative purposes, a simply structured fictitious example is considered. It is restricted to the number of $N=5$ actors. The numerical values are chosen so that the required calculations remain relatively easy. The calculations can also be carried out by computer using spreadsheet software such as Microsoft Excel ${ }^{\circledR}$.

Furthermore, the following example should illustrate what information is required in management practice in order to apply the $\tau$-value for the calculation of cooperation gain distributions. Finally, the fictitious example has the virtue of being usable as some kind of benchmark [29] for the comparison of game theory solution concepts for distribution problems, since it in principle uses the same data as in [7] and [25] to calculate the Shapley value and the Nucleolus as alternative game theory solution concepts for distribution problems. In this regard, the following benchmark example is in keeping with the pertinent literature.

It should be noted, however, that the example is not concerned with the gathering of information. In management practice, obtaining all values of the characteristic function $c$ for all possible coalitions could prove particularly difficult. This information gathering problem is not specific to the $\tau$-value, but concerns all solution concepts of cooperative game theory. This is the reason why the information gathering problem is not approached more closely in this paper.

The numerical example considers a value chain with 5 actors $A_{1}, \ldots, A_{5}$. In the last business year, the actors in the value chain jointly realized an cooperation gain $G$ to the amount of $\$ 100,000$. This cooperation gain is to be distributed among the five actors of the value chain in a manner that these actors accept as fair. First of all, to ensure the comparability with other game theory solution concepts like the Shapley value and the nucleolus, it is assumed that the values of the characteristic function $c$ for the generic distribution game are known completely. Thus the values $c\left(C_{m}\right)$ are known for every possible coalition $C_{m}$ which can be formed from the set of actors $A=\left\{A_{1}, \ldots, A_{5}\right\}$. These values $c\left(C_{m}\right)$ are given in Table 1 for all $2^{5}-1=31$ coalitions $C_{m}$ where $m=0,1,2, \ldots, 30$. 
Table 1. Values of the characteristic function $c$ for all coalitions $C_{m}$

\begin{tabular}{c|r|c|c|c|c}
\hline$C_{m}$ & $c\left(C_{m}\right)$ & \multicolumn{1}{|c|}{$C_{m}$} & $c\left(C_{m}\right)$ & \multicolumn{2}{|c}{$C_{m}$} \\
\hline$C_{0}=\left\{A_{1}, A_{2}, A_{3}, A_{4}, A_{5}\right\}$ & 100,000 & \multicolumn{5}{|l}{$c$} \\
\hline$C_{1}=\left\{A_{1}\right\}$ & 0 & $C_{11}=\left\{A_{2}, A_{4}\right\}$ & 25,000 & $C_{21}=\left\{A_{1}, A_{4}, A_{5}\right\}$ & 55,000 \\
\hline$C_{2}=\left\{A_{2}\right\}$ & 0 & $C_{12}=\left\{A_{2}, A_{5}\right\}$ & 30,000 & $C_{22}=\left\{A_{2}, A_{3}, A_{4}\right\}$ & 50,000 \\
\hline$C_{3}=\left\{A_{3}\right\}$ & 0 & $C_{13}=\left\{A_{3}, A_{4}\right\}$ & 30,000 & $C_{23}=\left\{A_{2}, A_{3}, A_{5}\right\}$ & 55,000 \\
\hline$C_{4}=\left\{A_{4}\right\}$ & 5,000 & $C_{14}=\left\{A_{3}, A_{5}\right\}$ & 35,000 & $C_{24}=\left\{A_{2}, A_{4}, A_{5}\right\}$ & 65,000 \\
\hline$C_{5}=\left\{A_{5}\right\}$ & 10,000 & $C_{15}=\left\{A_{4}, A_{5}\right\}$ & 45,000 & $C_{25}=\left\{A_{3}, A_{4}, A_{5}\right\}$ & 70,000 \\
\hline$C_{6}=\left\{A_{1}, A_{2}\right\}$ & 0 & $C_{16}=\left\{A_{1}, A_{2}, A_{3}\right\}$ & 25,000 & $C_{26}=\left\{A_{1}, A_{2}, A_{3}, A_{4}\right\}$ & 60,000 \\
\hline$C_{7}=\left\{A_{1}, A_{3}\right\}$ & 5,000 & $C_{17}=\left\{A_{1}, A_{2}, A_{4}\right\}$ & 35,000 & $C_{27}=\left\{A_{1}, A_{2}, A_{3}, A_{5}\right\}$ & 65,000 \\
\hline$C_{8}=\left\{A_{1}, A_{4}\right\}$ & 15,000 & $C_{18}=\left\{A_{1}, A_{2}, A_{5}\right\}$ & 40,000 & $C_{28}=\left\{A_{1}, A_{2}, A_{4}, A_{5}\right\}$ & 75,000 \\
\hline$C_{9}=\left\{A_{1}, A_{5}\right\}$ & 20,000 & $C_{19}=\left\{A_{1}, A_{3}, A_{4}\right\}$ & 40,000 & $C_{29}=\left\{A_{1}, A_{3}, A_{4}, A_{5}\right\}$ & 80,000 \\
\hline$C_{10}=\left\{A_{2}, A_{3}\right\}$ & 5,000 & $C_{20}=\left\{A_{1}, A_{3}, A_{5}\right\}$ & 45,000 & $C_{30}=\left\{A_{2}, A_{3}, A_{4}, A_{5}\right\}$ & 90,000 \\
\hline
\end{tabular}

A prerequisite for calculation of the $\tau$-value as a solution $\underline{v}_{\tau}$ with $\underline{v}_{\tau}=\left(v_{1 . \tau}, \ldots, v_{N . \tau}\right)$ for the generic distribution game is that the values of the characteristic function $c$ for all three types of coalitions are available. That is, $c\left(C_{0}\right)$ has to be available for the grand coalition $C_{0}=\left\{A_{1}, \ldots, A_{5}\right\}$, while $c\left(M C_{n}\right)$ is required for each marginal coalition $M C_{n}$ with $n=1, \ldots, 5$ and $c\left(A C_{n . q}\right)$ must be known for each outsider coalition $A C_{n . q}$.

The value $c\left(C_{0}\right)=100,000$ for the grand coalition $C_{0}$ is immediately available from Table 1 , since, according to the efficiency condition, the whole cooperation gain $G=100,000$ must be distributed exactly among all 5 actors $A_{1}, \ldots, A_{5}$ in the value chain.

The values $c\left(M C_{n}\right)$ for the marginal coalitions $M C_{n}$ with $n=1, \ldots, 5$ can be determined with the aid of the definition $M C_{n}=C_{0} \backslash\left\{A_{n}\right\}$. The results are shown in Table 2 .

Table 2. Values of the characteristic function $c$ for all marginal coalitions $M C_{n}$

\begin{tabular}{l|l}
\hline$M C_{n}$ & $c\left(M C_{n}\right)$ \\
\hline$M C_{1}$ & $c\left(\left\{A_{1}, \ldots, A_{5}\right\} \backslash\left\{A_{1}\right\}\right)=c\left(\left\{A_{2}, A_{3}, A_{4}, A_{5}\right\}\right)=90,000$ \\
\hline$M C_{2}$ & $c\left(\left\{A_{1}, \ldots, A_{5}\right\} \backslash\left\{A_{2}\right\}\right)=c\left(\left\{A_{1}, A_{3}, A_{4}, A_{5}\right\}\right)=80,000$ \\
\hline$M C_{3}$ & $c\left(\left\{A_{1}, \ldots, A_{5}\right\} \backslash\left\{A_{3}\right\}\right)=c\left(\left\{A_{1}, A_{2}, A_{4}, A_{5}\right\}\right)=75,000$ \\
\hline$M C_{4}$ & $c\left(\left\{A_{1}, \ldots, A_{5}\right\} \backslash\left\{A_{4}\right\}\right)=c\left(\left\{A_{1}, A_{2}, A_{3}, A_{5}\right\}\right)=65,000$ \\
\hline$M C_{5}$ & $c\left(\left\{A_{1}, \ldots, A_{5}\right\} \backslash\left\{A_{5}\right\}\right)=\mathrm{c}\left(\left\{A_{1}, A_{2}, A_{3}, A_{4}\right\}\right)=60,000$ \\
\hline
\end{tabular}

The values $c\left(A C_{n . q}\right)$ for the outsider coalitions $A C_{n . q}$ with $n=1, \ldots, 5$ can be obtained immediately from Table 1. However, the calculation of these values $c\left(A C_{n . q}\right)$ requires a tremendous amount of work, since $75\left(5 \bullet 15=n^{\bullet} q\right)$ feasible outsider coalitions have to be considered. This calculation is therefore omitted for space reasons (elaborate calculations can be found in [16, p. 219]). It is significant that for the calculation of the values $c\left(A C_{n . q}\right)$ for all combinatorial possible outsider coalitions $A C_{n . q}$, the values of the characteristic function $c$ for all possible coalitions $C_{m}$ with $\varnothing \subset C_{m} \subset C_{0}$ need to be determined. Hence the requirement of minimal knowledge is not fulfilled by the $\tau$-value. This is surprising, because it seems from the formulas (2.1) to (2.6) that determine the $\tau$-value, only those values of the characteristic function $c$ must be known that refer to the grand coalition $C_{0}$, the marginal coalitions $M C_{n}$ and the outsider coalitions $A C_{n . q}$. Only 
the concrete numeric calculation of the $\tau$-value for the example considered here shows that the calculation of the values $c\left(A C_{n . q}\right)$ for the outsider coalitions $A C_{n . q}$ indirectly leads to the fact that the values $c\left(C_{m}\right)$ of the characteristic function $c$ for all possible coalitions $C_{m}$ with $\varnothing \subset C_{m} \subset C_{0}$ must be known.

The components $v_{n \text { max }}$ of the upper bound $U B$ (ideal point) are calculated with formula (2.2.3) on the basis of the values $c\left(C_{0}\right)$ and $c\left(M C_{n}\right)$. The value $c\left(C_{0}\right)$ is immediately given by the efficiency gain $G$ to be distributed: $c\left(C_{0}\right)=G=100,000$. Thus the components $v_{n \text {.max }}$ of the upper bound $U B$ of the $\tau$-value are those in Table 3 .

Table 3. Components $v_{n \text {.max }}$ of the upper bound $U B$ of the $\tau$-value

\begin{tabular}{l|l}
\hline$A_{n}$ & $v_{n, \max }$ \\
\hline$A_{1}$ & $c\left(C_{0}\right)-c\left(M C_{1}\right)=100,000-90,000=10,000$ \\
\hline$A_{2}$ & $c\left(C_{0}\right)-c\left(M C_{2}\right)=100,000-80,000=20,000$ \\
\hline$A_{3}$ & $c\left(C_{0}\right)-c\left(M C_{3}\right)=100,000-75,000=25,000$ \\
\hline$A_{4}$ & $c\left(C_{0}\right)-c\left(M C_{4}\right)=100,000-65,000=35,000$ \\
\hline$A_{5}$ & $c\left(C_{0}\right)-c\left(M C_{5}\right)=100,000-60,000=40,000$ \\
\hline
\end{tabular}

The components $v_{n \cdot \min }$ of the lower bound $L B$ (threat point) of the $\tau$-value are calculated with formula (2.2.5) for each of the five actors $A_{1}$ to $A_{5}$. This calculation is shown as an example for $A_{4}$ :

$v_{4, \min }=\max \left\{c_{4.1} ; c_{4.2} ; 0\right\}=\max \{5,000 ; 5,000 ; 0\}=5,000$

because:

$c_{4.1}=c\left(\left\{A_{4}\right\} \mid A C_{4.1}\right)=c\left(\left\{A_{4}\right\}\right)=5,000$

$c_{4.2}=\max \left\{c\left(\left\{A_{4}\right\} \mid A C_{4 . q}\right)=c\left(A C_{4 . q}\right)-\sum_{m \in\left(N_{4, q} \backslash\{4\}\right)} v_{m . \max } \mid q=2, \ldots, 15\right\}=5,000$

It follows from the components $v_{n \text { max }}$ of the upper bound $U B$ and from the components $v_{n . \min }$ of the lower bound $L B$ calculated above that the standard case for the calculation of the $\tau$-value with $\sum_{n=1}^{N} v_{n \text {.max }} \neq \sum_{n=1}^{N} v_{n \text {.min }}$ applies. According to formula (2.2.9) the weighting factor $\gamma$ is as follows:

$$
\begin{aligned}
\gamma & =\frac{G-\sum_{n=1}^{N} v_{n . \text { min }}}{\sum_{n=1}^{N} v_{n, \max }-\sum_{n=1}^{N} v_{n, \text { min }}} \\
& =\frac{100,000-(0+0+0+5,000+10,000)}{(10,000+20,000+25,000+35,000+40,000)-(0+0+0+5,000+10,000)} \\
& =\frac{17}{23} \approx 0,74
\end{aligned}
$$

The components $v_{n . \tau}$ of the $\tau$-value $\underline{v}_{\tau}$ are then calculated in Table 4 for each actor $A_{n}$ with the help of formula (2.2.8) and the weighting factor $\gamma={ }^{17} / 23$ as the convex combination of the com 
ponents $v_{n . \max }$ of the upper bound $U B$ (threat point) and the components $v_{n . \min }$ of the lower bound $L B$ (threat point) for the $\tau$-value.

Table 4. Components $v_{n . \tau}$ of the $\tau$-value $\underline{v}_{\tau}$

\begin{tabular}{l|l}
\hline$A_{\mathrm{n}}$ & $v_{n . \tau}$ \\
\hline$A_{1}$ & $17 / 23 \cdot 10,000+6 / 23 \bullet 0=1 / 23 \cdot 170,000$ \\
\hline$A_{2}$ & $17 / 23 \cdot 20,000+6 / 23 \bullet 0=1 / 23 \cdot 340,000$ \\
\hline$A_{3}$ & $17 / 23 \cdot 25,000+6 / 23 \cdot 0=1 / 23 \cdot 425,000$ \\
\hline$A_{4}$ & $17 / 23 \cdot 35,000+6 / 23 \cdot 5,000=1 / 23 \cdot 625,000$ \\
\hline$A_{5}$ & $17 / 23 \cdot 40,000+6 / 23 \cdot 10,000=1 / 23 \cdot 740,000$ \\
\hline
\end{tabular}

In the end, there is exactly one $\tau$-value $\underline{v}_{\tau}={ }^{1} / 23 \cdot(170,000 ; 340,000 ; 425,000 ; 625,000 ; 740,000)$ as a unique solution for the special distribution problem considered here as an example.

\section{CONCLUSIONS}

This article has shown how the vague understanding of fairness that dominates in practice can be operationalized with the aid of game theory by applying a game theory solution concept to the generic distribution problem. Special importance was attached to the fact that the $\tau$-value inevitably results if a small number of assumptions with respect to individual and collective rationality, efficiency and integrity is accepted. This matches the justification program introduced at the beginning and presents a game theory solution concept in which good reasons are cited in order for the resulting solutions to be accepted as fair distribution.

The authors consider the assumptions that need to be accepted in order to use the $\tau$-value to be so weak that this solution concept has great potential for general acceptance. Other game theory solution concepts, for example the Shapley value and the nucleolus, demand the acceptance of far more abstract, often only formally precisely definable assumptions. Hence they have considerably lower general acceptance potential. Additionally, other game theory solution concepts, for example the core of a game, can be traced back to a few plausible assumptions. However, they have the disadvantage that they do not exist for many instances of the generic distribution problem or have multiple, often even an infinite number of solutions. From a practical perspective, neither is acceptable. For the aforementioned reasons, the $\tau$-value both offers to unite the advantage of good reasons for the acceptability of distribution outcomes as fair with the pragmatic assumptions of the existence and uniqueness for a - in relation to other game theory solution concepts - broad range of instances of the generic distribution problem.

As managerial insights three aspects can be gained from the explanations of the chapters above.

Firstly, game theory solution concepts such as the $\tau$-value offer a "reasonable", because provable with good reasons, and justifiable basis for the distribution of cooperation gains in value chains. Thanks to the explicability of the good reasons, there is a high chance that the companies cooperating in a value chain will accept the distribution of the collectively realized cooperation gains as fair. However, the distribution of a cooperation gain determined with the aid of the $\tau$-value can always only represent the source of a discussion about the fair distribution of a collectively realized cooperation gain, and not the final outcome of the distribution. Because the $\tau$-value is, like any other concept for distributing cooperation gains, based on a few specific assumptions, it can, but must not, be accepted as "plausible" or "reasonable". Propositions for the 
distribution of cooperation gains on the basis of the $\tau$-value indeed form a promising basis for discussion, because such a distribution proposition can be justified with good reasons. However, good reasons never offer an assurance that - especially on the basis of other assumptions - even more convincing reasons for an alternative distribution proposition can be found.

Secondly, it was presupposed in this contribution, that the cooperation gain $G$ can be defined precisely and quantified monetarily, but this assumption will only rarely be fulfilled in practice. Hence for adaptability of the game theory solution concept presented here, the management of the value chain needs to ensure that the cooperation gain to be distributed can also be defined in concrete terms. This can lead to two basic practical problems. On the one hand, agreement needs to be reached inside a value chain as to the concrete economic scale on which the cooperation gain to be distributed is to be determined and from which sources the information required to determine it can be drawn. This is not a trivial task and cannot be analyzed in detail in this article. On the other hand, how the management of a value chain is defined needs to be clarified, because a value chain according to the agreements made at the beginning is characterized by the cooperation of legally autonomous companies (autonomous actors). If a value chain is dominated by a focal company, the management of a value chain can be equated in a relatively simple way with the management of the focal company. However, as a side condition it must be considered that the management of the focal company can only make decisions that do not jeopardize the stability of the value chain - and from a game theory perspective the stability of the grand coalition. There is also the question of what the management of a value chain is if the special case of a focal company does not apply. In this non-focal case, one option is to revert to the game theory concept of coalition formation games. With the aid of this concept, it is possible to examine how coalitions of legally autonomous companies in the form of a value chain come about. However, even such coalition formation games so far offer no starting points at which to determine how in value chains without a focal company the cooperation gain to be distributed should be determined in concrete terms. Extensive academic research is still required on this point.

Thirdly, the management of companies that cooperate in a value chain must always be aware of the fact, that game theory solution concepts assume the rationality of all involved actors (companies). This rationality of actors manifests itself mainly in the conditions of individual and collective rationality. However, it is also based on assumptions such as the efficiency condition that can also be seen as an expression of collective rationality. Negotiations in real existing value chains about the "fair" distribution of cooperation gains are by no means always affected by the rationality of the negotiating partners. Rather, the management must be aware that the process of negotiation on the fair distribution of cooperation gains is also influenced by the fact that the rationality of actors does frequently not correspond to classic game theory. Such influences can extend from the many determinants of the so-called bounded rationality that is increasingly attracting interest in economic analysis, up to a large number of effects of apparently or even real irrational behavior. All these influences "beyond" the conception of classic rationality are not covered by the game theory solution concept introduced here. Hence the management of companies that cooperate in a value chain must always be aware that the game theory solution concept considered here only covers one part of the real process of negotiation over fair distributions of cooperation gains.

The $\tau$-value has the advantage, that it can be referred with the aid of the new interpretation presented in chapter 3.2, directly to the bargaining power of the actors cooperating in a value chain. It has been shown, that the $\tau$-value immediately results from the positive and the negative network effects that an actor can contribute to the realization of a cooperation gain in a value chain. Hence the $\tau$-value represents an interesting approach, which allows the aspect of the bargaining power to be included in the determination of distribution outcomes that can be accepted as fair. 
One problem in the determination of the $\tau$-value needs to be mentioned that is a reason for further research. It results from violation of the requirement of minimal knowledge. Knowledge of the values of the characteristic function $c$ for all possible coalitions is also needed for the $\tau$ value. This represents a serious barrier to the implementation of the $\tau$-value in practice, because normally it cannot be assumed, that enough knowledge and enough time are available to determine the concrete function values $c\left(C_{m}\right)$ for all possible coalitions $C_{m}$. Hence "intelligent" approaches are needed, to reduce the effort involved in their determination. It should incidentally be mentioned that this problem of the prohibitive amount of effort required to determine the concrete values of the characteristic function for all possible coalitions does not represent a specific problem for the solution concept of the $\tau$-value introduced here. It actually concerns all common game theory solution concepts such as the Shapley value and the nucleolus.

But considering the $\tau$-value, it is not necessary to consider all coalitions $C_{m}$ with $\varnothing \subset C_{m} \subset C_{0}$ for the calculation of the function values $c\left(A C_{n . q}\right)$ of the characteristic function $c$ for the outsider coalitions $A C_{n . q}$. A first approach has already been developed [16, pp. 233-236 and 244-255]. It is based on the pragmatic thought, that experienced managers often know, that an outsider coalition $A C_{n . q}$ is insignificant, because the actor $A_{n}$, who leads this outsider coalition, cannot pose a credible threat with the significant amount $c\left(A C_{n . q}\right)>0$, that he or she could realize in this outsider coalition. Additionally, an experienced manager can know that an actor $A_{n}$ is not relevant to the calculation of the $\tau$-value, because this actor $A_{n}$ cannot lead any outsider coalition $A C_{n . q}$ with a credible threat of the amount $c\left(A C_{n . q}\right)>0$. If this knowledge about insignificant outsider coalitions $A C_{n . q}$ and not relevant actors $A_{n}$ can be considered additionally, the effort required to calculate the $\tau$-value can be reduced significantly. This requires a few algorithmic modifications regarding the concrete calculation of $\tau$-value. These changes will be discussed in detail in a later publication.

\section{REFERENCES}

[1] Li, S., Zhu, Z. \& Huang, L. (2009). "Supply chain coordination and decision making under consignment contract with revenue sharing". International Journal of Production Economics, Vol. 120, No. 1, pp. 88-99.

[2] Mahdavi, I., Mohebbi, S., Cho, N., Paydar, M. M. \& Mahdavi-Amiri, N. (2008). "Designing a dynamic buyer-supplier coordination model in electronic markets using stochastic Petri nets". International Journal of Information Systems and Supply Chain Management, Vol. 1, No. 3, pp. 1-20.

[3] Saharidis, G. K. D., Kouikoglou, V. S. \& Dallery, Y. (2009). “Centralized and decentralized control policies for a two-stage stochastic supply chain with subcontracting". International Journal of Production Economics, Vol. 117, No. 1, pp. 117-126.

[4] Xiao, T., Luo, J. \& Jin, J. (2009). "Coordination of a supply chain with demand stimulation and random demand disruption”. International Journal of Information Systems and Supply Chain Management, Vol. 2, No. 1, pp. 1-15.

[5] Zhang, D. (2006). "A network economic model for supply chain versus supply chain competition”. Omega - The International Journal of Management Science, Vol. 34, No. 3, pp. 283-295.

[6] Cachon, G. P. \& Zipkin, P. H. (1999). "Competitive and Cooperative Inventory in a Two-Stage Supply Chain”. Management Science, Vol. 45, No. 7, pp. 936-953.

[7] Fromen, B. (2004). Fair Distribution in Enterprise Networks - Solution Concepts Stemming from Cooperative Game Theory (in German). Doctoral dissertation, University of DuisburgEssen, Germany. Wiesbaden: Gabler. 
International Journal of Managing Value and Supply Chains (IJMVSC) Vol. 2, No. 1, March 2011

[8] Gjerdrum, J., Shah, N. \& Papageorgiou, L. G. (2001). “Transfer Prices for Multienterprise Supply Chain Optimization”. Industrial \& Engineering Chemistry Research, Vol. 40, No. 7, pp. $1650-1660$

[9] Inderfurth, K. \& Minner, S. (2001): "Production and Logistics" (in German). In P.-J. Jost (Ed.), Spieltheorie in der Betriebswirtschaftslehre, pp. 307-349. Stuttgart: Schäffer-Poeschel.

[10] Minner, S. (2007). "Bargaining for cooperative economic ordering”. Decision Support Systems, Vol. 43. No. 2, pp. 569-583.

[11] Sucky, E. (2004). Coordination in supply chains: Game theoretical analysis for the determination of integrated procurement and production policies (in German). Doctoral dissertation, University of Frankfurt am Main 2003. Wiesbaden: Gabler.

[12] Sucky, E. (2004). "Coordinated order and production policies in supply chains". OR Spectrum, Vol. 26, No. 4, pp. 493-520.

[13] Sucky, E. (2005). "Inventory management in supply chains: A bargaining problem". International Journal of Production Economics, Vol. 93-94 (Proceedings of the Twelfth International Symposium on Inventories), pp. 253-262.

[14] Thun, J.-H. (2005). “The Potential of Cooperative Game Theory for Supply Chain Management”. In H. Kotzab, S. Seuring, M. Müller \& G. Reiner (Eds.), Research Methodologies in Supply Chain Management, pp. 477-491. Heidelberg et al.: Springer.

[15] Voß, S., \& Schneidereit, G. (2002). "Interdependencies between Supply Contracts and Transaction Costs". In S. Seuring \& M. Goldbach (Eds.), Cost Management in Supply Chains, pp. 253272. Heidelberg: Physica.

[16] Zelewski, S. (2009). Fair distribution of efficiency gains in supply webs: a game theoretic approach based on the $\tau$-value (in German). Berlin: Logos.

[17] Tijs, S. H. (1981). "Bounds for the core and the $\tau$-value". In O. Moeschlin \& D. Pallaschke (Eds.), Game Theory and Mathematical Economics, pp. 123-132. Amsterdam: North Holland Publishing Company.

[18] Driessen, T. S. H. (1985). Contributions to the Theory of Cooperative Games: The $\tau$-Value and $k$-convex Games. Unpublished doctoral dissertation, University of Nijmegen, The Netherlands.

[19] Driessen, T. \& Tijs, S. (1982). "The $\tau$-value, the nucleolus and the Core for a subclass of games”. In H. Loeffel \& P. Stähly (Eds.), Methods of Operations Research 46, pp. 395-406. Königstein: Verlagsgruppe Athenäum Hain Hanstein.

[20] Driessen, T. S. H. \& Tijs, S. H. (1983). Extensions and Modifications of the $\tau$-Value for Cooperative Games (Report No. 8325). Nijmegen, The Netherlands: University of Nijmegen, Department of Mathematics.

[21] Driessen, T. S. H. \& Tijs, S. H. (1985). "The $\tau$-value, the Core and semiconvex games". International Journal of Game Theory, Vol. 14, No. 4, pp. 229-247.

[22] Tijs, S. H. (1987). “An axiomatization of the tau-value”. Mathematical Social Sciences, Vol. 13, No. 2, pp. 177-181.

[23] Tijs, S. H. \& Driessen, T. S. H. (1983). The $\tau$-Value as a Feasible Compromise Between Utopia and Disagreement (Report 8312). Nijmegen, The Netherlands: University of Nijmegen, Department of Mathematics.

[24] Tijs, S. H. \& Driessen, T. S. H. (1986). "Game theory and cost allocation problems". Management Science, Vol. 32, No. 8, pp. 1015-1028.

[25] Curiel, I. (1997). Cooperative Game Theory and Applications - Cooperative Games Arising from Combinatorial Optimization Problems. Boston: Kluwer Academic Publishers. 
[26] Kuhn, H. W., Harsanyi, J. C., Selten, R., Weibull, J. W., Damme, E. van Nash, J. F. Jr., \& Hammerstein, P. (1996). "The work of John Nash in game theory - Nobel seminar, December 8, 1994”. Journal of Economic Theory, Vol. 69, No. 1, pp. 153-185.

[27] Bilbao, J. M., Jiménez-Losada, A., Lebrón, E. \& Tijs, S. H. (2002). “The $\tau$-value for games on Matroids". Top, Vol. 10, No. 1, pp. 67-81.

[28] Branzei, R., Dimitrov, D. \& Tijs, S. (2005). Models in Cooperative Game Theory - Crisp, Fuzzy, and Multi-Choice Games. Berlin: Springer.

[29] Ajelabi, I. \& Tang, Y. (2010). "The Adoption of Benchmarking Principles for Project Management Performance Improvement”. International Journal of Managing Public Sector Information and Communication Techniques, Vol. 1, No. 2, pp. 1-8.

\section{Authors}

Prof. Dr. Stephan Zelewski teaches business management with focus on production management at the University of Duisburg-Essen. He holds the chair for Production and Industrial Information Management at the Faculty of Economics at the campus of Essen. $\mathrm{He}$ also is member of the Institute of Business and Economic Studies (IBES), the Institute for Computer Science and Business Information Systems (ICB) as well as the Centre for Logistics \& Traffic (ZLV). His work scope contains production management, especially logistics and supply chain management, the use of modern computer technologies in the area of production management, operations research and game theory, knowledge management and artificial intelligence and their operational applications (especially knowledge based systems and multi-agent systems), production theory as well as philosophy of science. For further information please visit: http://www.pim.wiwi.uni-due.de/team/stephan-zelewski/.

Dipl. Wirt.-Inf. Susanne Jene is a research assistant at the chair for Production and Industrial Information Management at the Faculty of Economics at the University of Duisburg-Essen. Her work scope contains production management, especially cooperative game theory, business networks, supply chain management and enterprise resource planning systems as well as project management. For further information please

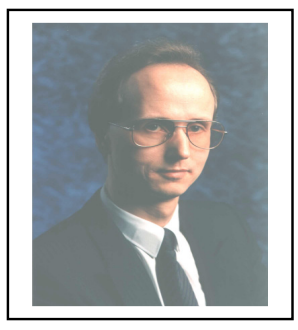
visit:http://www.pim.wiwi.unidue.de/team/susanne-jene/.

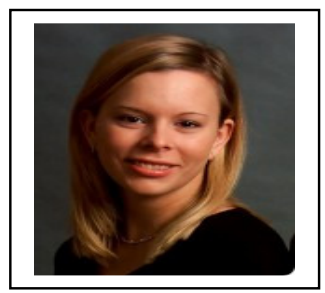

